\title{
Analysis of power line communications for last-hop backhaul in small cells deployment
}

\author{
José A. Cortés*, Francisco J. Cañete*, Matías Toril*, Luis Díez*, Alicia García-Mozos ${ }^{\dagger}$ \\ ${ }^{*}$ Universidad de Málaga, E.T.S.I. Telecomunicación, Dpt. Ingeniería Comunicaciones, \\ email: \{jaca, francis, mtoril, diez\}@ic.uma.es \\ $\dagger$ Vodafone Group, email: aligmozos@gmail.com
}

\begin{abstract}
The purpose of this work is to study the feasibility of using power line communications (PLC) over outdoor public lighting networks (OPLN) for lasthop backhaul in small cell deployment. The analysis is based on actual noise measurements performed in two OPLN in the city of Málaga (Spain) and on a bottom-up channel simulator, which has been designed according to the physical characteristics and the common practices in such kind of networks. Estimations of the bitrate achieved by PLC systems following the ITU-T Rec. G.9960 (G.hn) standard, are performed and discussed. Results indicate that PLC is a promising solution for this application.
\end{abstract}

Index Terms-Small cells, backhaul, power line communications, outdoor public lighting networks.

\section{INTRODUCTION}

The increasing traffic demand in mobile cellular systems can be tackled in three ways: by using more spectrally efficient systems, by increasing the available spectrum and by increasing the number of cells. Since the first and second ways are not always possible, only the last one is a feasible alternative in most cases. However, the traditional macro-cellular deployment alone is not an option any more in a high-demand dataoriented cellular scenario where it would result in both capital and operational inefficiencies. Hence, low-cost and low-power base transceiver stations (BTS) with coverage range from tenths to several hundred meters, named small cells, are also employed [1]. Small cells appear as a key enabler to complement macro architectures and provide effective network planning to cope with bandwidth-intensive applications and services using limited radio resources, especially in dense spots and cell edges [2].

Dense deployments of small cells demand costefficient solutions for the backhaul (the link that connects the cell sites to the first point of concentration) that provide the required reliability, latency and data rates. While the latter depend on the cell site technology and configuration, typical values for Long Term Evolution (LTE) small cells are about $50 \mathrm{Mbit} / \mathrm{s}$ [3]. The two main wireless technologies used for this backhaul are free-space optical (FSO) and wireless radio, but none of them provide a constraint-free solution. While non-line-of-sight (NLOS) technologies imply high operational cost and interference, line-of-sight (LOS) alternatives might not always be a feasible option in high-dense urban deployments. Moreover, the 978-1-7281-0289-4/19/\$31.00 (c)2019 IEEE lack of available spectrum for microwave backhauling is becoming a serious problem in certain locations [4]. Regarding FSO, while it could be an attractive technology, its cost is still too high.

In this context, the use of power line communications (PLC) for the backhaul is promising since offers important advantages compared to current wired technologies like fiber optics or digital subscriber lines, namely the ubiquity of the low voltage (LV) distribution network and the fact that no new installations are required. Models of the traffic to be backhauled and the power line topology feeding the cells base stations has already been studied in [5]. This work focuses on the use of PLC over outdoor public lighting networks (OPLN). Since the height of outdoor lamp posts range 3 to $14 \mathrm{~m}$, they can be used to hold small cells BTS, which are usually placed at 4 to $7 \mathrm{~m}$ height, improving the system capacity in high streets, central squares and the surroundings of bus and train stations, etc.

The paper is organized as follows. In the second section, the main characteristics of the OPLN are presented and, in the third one, network scenarios are discussed. The fourth section deals with the characterization and modeling of the channels, whereas the estimated performance of PLC systems is studied in the fifth section. Finally, some conclusions are given.

\section{Structure OF OPLN}

OPLN can be defined as the last part of the power distribution network and consist of the control panel, the different output lines organized in a series of circuits and the lamps. The input to the control panel are independent power lines coming from the distribution transformer. Circuits usually contain three conductors for the phases (usually denoted as R, S and $\mathrm{T}$ ) and an additional one for the common neutral. Both 4pole and monopolar cables (deployed inside a common conduit) are employed. Lamps are distributed in each circuit by alternately assigning them to one of the three phases. A simplified example of such networks, with just one circuit, is shown in Fig. 1. Control panels are commonly located in the sidewalk and contain metering devices (overload protection devices, power control switchers, thermal magnetic circuit breakers, differential circuit breakers, etc.) and control devices (like manually-operated switches and daily time controlled switches). Activation of lighting is done by 
either a central controller or an astronomical clock that computes sunrise/sunset times on a daily basis.

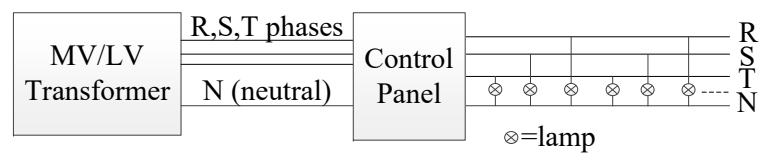

Fig. 1. Simplified structure of OPLN.

In order to decrease the power consumption, a luminous Flux Reducer Stabilizer (FRS) is usually employed in OPLN. The FRS is a large device installed beside the control panel that reduces the mains voltage level from $230 \mathrm{~V}$ to $180 \mathrm{~V}$ (approximately). Hence, in the deployment, both the wireless base stations and the PLC modems must be able to operate with this lower supply voltage (unless an additional ad-hoc supply circuit is installed to feed them).

A tree-like topology is often adopted for the deployment of OPLN, with a more linear or branched shape depending on street characteristics. In addition, the topology of OPLN is also subject to some electrical constraints. For instance, the maximum length of the circuit cables must guarantee that the voltage drop at the line end does not exceed a given percentage, e.g., 3\% in Spain. Moreover, the number of connected conductors in the cables diminish towards the end of a circuit. Generally, there are only two active conductors, i.e. connected to the mains, in the cable that departures from the ante-penultimate lamp post base to the penultimate lamp post and only one from the base of the penultimate lamp post to the last one.

\section{NETWORK SCENARIOS}

In this section, the OPLN used in the performance assessment are presented. The strategy to design such networks is focused on physical and electrical parameters (e.g., distances, cable sections, cable materials, loads, etc.) rather than on luminous criteria, although the latter are indirectly considered. The scenarios have been classified in:

1) Worst case scenarios. This set comprises the worst topologies that PLC systems may have to face and are useful to identify lower bounds in the performance.

2) Randomly created scenarios. In order to have a large number of channels with a reasonable dispersion in their behavior, a random network generator has been developed for this purpose.

3) Real scenarios. This group is formed by a number of topologies provided by the Servicios Operativos of the City Council of Málaga (Spain). They serve to assess PLC system performance in realistic topologies.

\section{A. Worst-case topologies}

From the channel response perspective, the worst conditions can be associated to very high attenuation levels. The latter may be due to long links, because of losses in the conductors by skin effect (incremental with frequency) that lead to low-pass channel responses, and to a large number of derivations from the main path from transmitter to receiver, which cause notches in the channel frequency response related to multipath propagation. Two kinds of networks have been considered in the study to represent both conditions: a long linear topology and a highly branched topology.

1) Linear topology

A 2-lane street has been chosen for this case. An example of its electrical system structure is shown in Fig. 2. Two types of linear topologies with this shape have been considered, one with 40 lamps and another with 99 lamps and shorter branches.

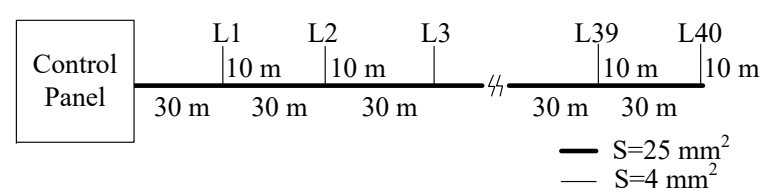

Fig. 2. Diagram of a linear network under test.

2) Highly branched topology

A Manhattan-like scenario, whose dimensions have been taken from the Ensanche district in Barcelona (Spain), has been selected for this case. The resulting network layout is represented in Fig. 3.

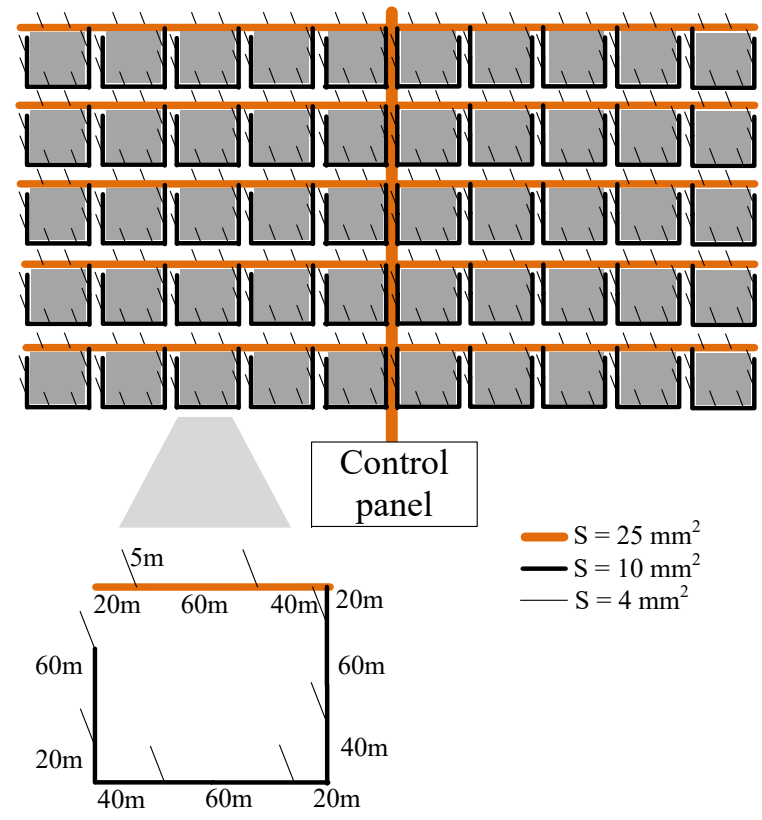

Fig. 3. Diagram of the highly branched network under test.

\section{B. Random networks}

A random network generator has been designed to create an ensemble of feasible OPLN topologies. It takes into account both physical and electrical parameters and the main design rules used in common practice: streets width, lighting deployment style (oneside, two-sides staggered or aligned, etc.), lamp-post separation, lamps power, cable section, etc. 
The core of the simulator is the Galton-Watson branching process [6]. In our implementation, the network is treated as a tree, divided into a number of hierarchical levels (defined by the user). The tree is generated from the root node (control panel) to the leaf nodes (lamps). At a certain level, each branch (segment) has a given probability to generate a number of branches. Once the tree network structure is built, a random distance is assigned to each branch. Such a distance ranges from 0 to the maximum distance of a straight segment (i.e., without branches) still satisfying a voltage drop constraint. Six types of random topologies have been defined according to the number of lamps: $\{15,19,21,22,26,27\}$. Realizations have been selected for each type (after multiple trials with this simulator) with a criterion of maximum diversity, i.e. by taking care of selecting those with rather different topologies.

\section{Real network scenarios}

To complete the set, several network scenarios have been constructed from the schemes provided by the local authority of Málaga. Two scenarios have been considered in this study: one corresponding to Aurora avenue (with a more linear structure) and other to the SOHO district (with a more branched structure) ${ }^{1}$.

\section{Channel CHARACTERIZATION AND MODELING}

A hybrid strategy has been followed in this study to estimate the PLC channel characteristics. The channel response is estimated using a bottom-up modelling approach based on multiconductor transmission line (MTL) theory [7]. This allows to estimate the response of a particular channel by knowing the network topology and can be used for network planning. However, the noise has been obtained by performing measurements in actual OPLN, since, to the best knowledge of the authors, neither published models nor empirical results are available in the literature for this scenarios.

\section{A. MTL modeling of OPLN}

As mentioned, OPLN are three-phase power networks in which lamps are connected between one phase and the neutral conductor. Fig. 4 depicts an example of simplified layout with two circuits. Thick lines indicate electrically-long wires for the considered frequency band. The transmitter is placed in phase $\mathrm{R}$ of circuit 1 and the receiver in phase $R$ of circuit 2 . The FRS is not shown.

From a communication point of view, OPLN can be modeled as a set of interconnected sections of MTL [8]. Each circuit consists of a four-conductor transmission line (TL) backbone from which either fourconductor TL derivations (branches) or two-conductor TL derivations (to the lamps), are deployed. Lamps are modeled with a real impedance whose values depend on their power consumption. The transmitter

\footnotetext{
1 See corresponding maps at https://goo.gl/tNdcgF and https://goo.gl/DeRqNA, respectively.
}

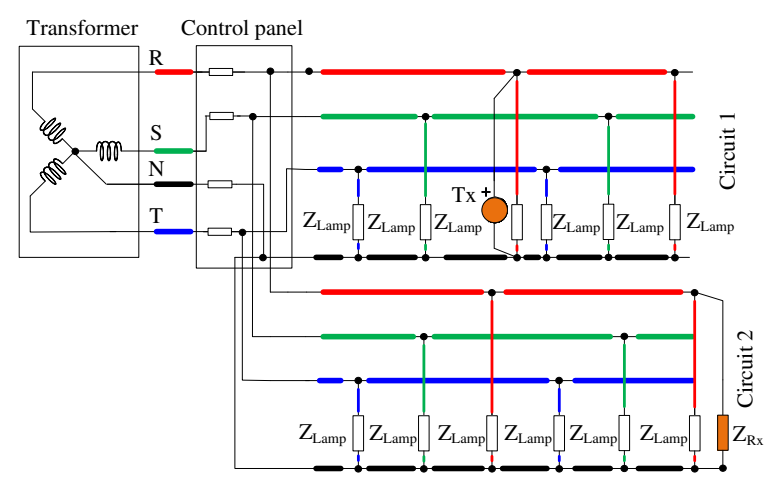

Fig. 4. Simplified structure of an outdoor public lighting network.

and receiver are also connected to the network by means of two-conductor TL. To calculate the response, an $(n+1)$-conductor TL segment ( $n$ conductors plus the neutral) can be modeled as a $2 n$-port network and cascaded multi-ports can be solved by means of matrix algebra.

\section{1) Example of modeled channel responses}

An example of some generated channels is given to illustrate the main characteristics of the frequency response. Fig. 5 shows the amplitude response of channels established over the modeled network corresponding to the Aurora avenue scenario, with link lengths that range from $20 \mathrm{~m}$ to $260 \mathrm{~m}$. As seen, channels exhibit a remarkable low-pass behavior due to the conductor losses. This contrasts to indoor PLC networks, where involved distances are relatively short and attenuation is mainly due to the dispersion caused by the high number of branches. Despite this, the ups and downs of the response due to the derivations effect is still noticeable, particularly in shorter links. However, deep notches can only be observed in the low frequency range (below $10 \mathrm{MHz}$ ), where the magnitude of the attenuation is still not so important and the effect of the multipath phenomenon can be observed.

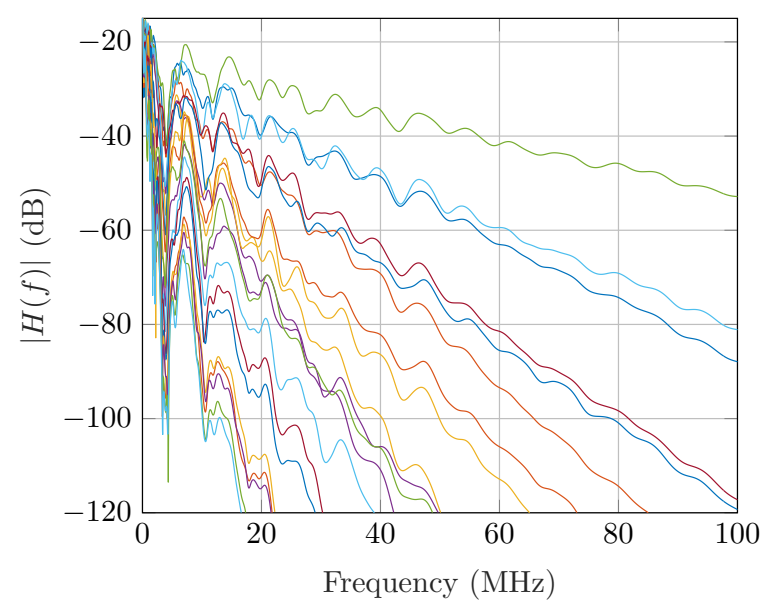

Fig. 5. Amplitude of channels established in the Aurora Av. topology. 


\section{2) Statistical analysis of channel characteristics}

The results of a statistical analysis accomplished over the overall set of channels are presented. This set comprises a mixture of channels generated on different scenarios: worst case ones, linear $(n=281$ channels) and branched $(n=176)$; realistic ones, SOHO $(n=190)$ and Aurora av. $(n=231)$; and the ones with randomly generated networks of different sizes $^{2}$ ( $\left.n=883\right)$. The total number of channels is 1741. The behavioral parameters estimated from the responses are the average attenuation and the delay spread [9]. The frequency band from $2 \mathrm{MHz}$ up to 80 $\mathrm{MHz}$, used by systems compliant with the ITU-T Rec. G.9906 standard [10], is analyzed. However, channel characteristics in the band $2-37.5 \mathrm{MHz}$, which is used by some traditional PLC modems, are provided as well [11].

Fig. 6 shows a scatter plot of the average attenuation vs the link length obtained for the two bands ${ }^{3}$. The regression lines fitting are also shown. Their expressions are $\alpha(\mathrm{dB})=0.75 L+16.5$, for the whole band, and $\alpha(\mathrm{dB})=0.442 L+15.8$, for the lower band, where $\alpha(\mathrm{dB})$ denotes the attenuation and $L$ is the link length in meters. These attenuation values are higher than those of indoor PLC channels, even for link lengths shorter than $100 \mathrm{~m}$. Since the large attenuation values of most channels longer than $300 \mathrm{~m}$ would make impossible to use them for communication purposes, they have been disregarded in the subsequent statistics.

The cumulative distribution function (CDF) of both the average attenuation and the delay spread are plotted in Fig. 7. In the frequency band $2-80 \mathrm{MHz}$, only in $20 \%$ of the channels have average attenuation lower than $40 \mathrm{~dB}$, while the median value is about $55 \mathrm{~dB}$. In the $2-37.5 \mathrm{MHz}$ band, the median value is about $50 \mathrm{~dB}$. Regarding the frequency selectivity, obtained values of the delay spread are about one half of the ones measured in indoor PLC networks [9], with a median value around $66 \mathrm{~ns}$ in the $2-80 \mathrm{MHz}$ band and $174 \mathrm{~ns}$ in the $2-30 \mathrm{MHz}$ one. Since current PLC systems have been designed for larger delay spreads, e.g., the ITU-T Rec. G.9960 employs a cyclic prefix of $5.6 \mu \mathrm{s}$, the maximum bit-rate will be limited by attenuation and not by channel distortion.

Finally, it must be noted that none of the links used in this study has the transmitter or receiver at the control panel. This point must be avoided because the noise is extremely high there (as it will be shown below) and the FRS has a very low impedance.

\section{B. Noise measurements}

PLC noise has been studied both in the context of indoor systems [12-14] and LV distribution networks [15] but, to the best of the authors' knowledge, not

\footnotetext{
${ }^{2}$ This is the larger subset because they are supposed to be more representative of actual networks.

${ }^{3}$ No channels with link lengths above $175 \mathrm{~m}$ (approximately) are shown due to the maximum attenuation values given by the Channel Generator. Beyond this point, average channel attenuation for longer channels is considered $\infty$.
}

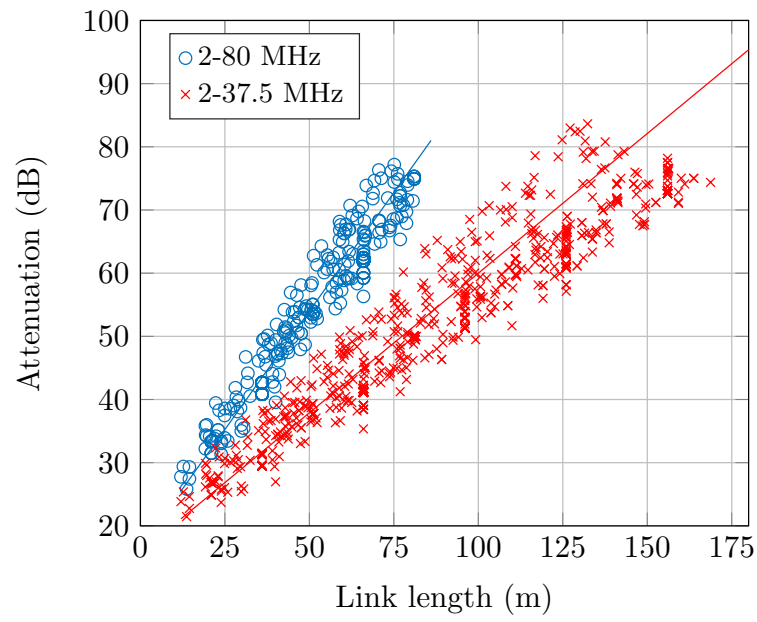

Fig. 6. Scatter plot of the average attenuation vs link length.

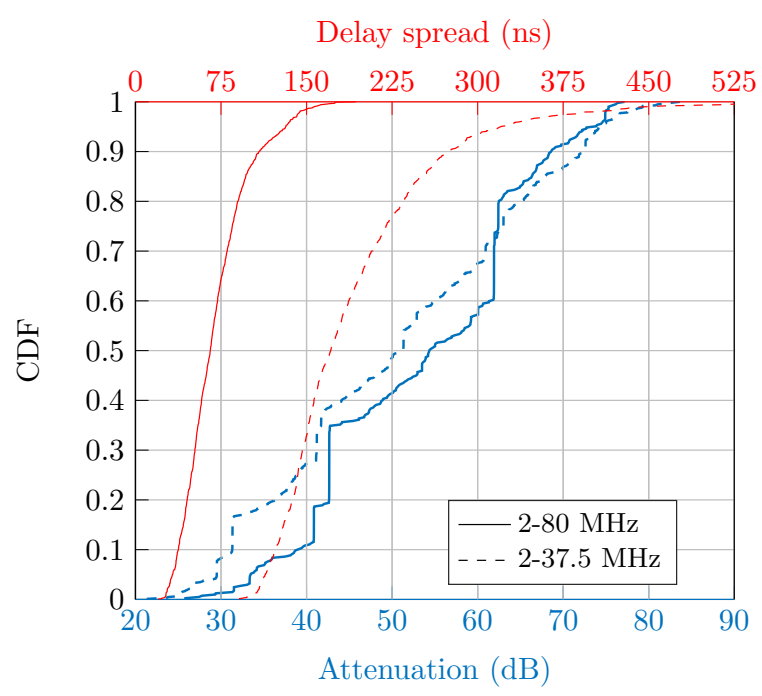

Fig. 7. CDF of both the average attenuation and delay spread.

in this kind of scenario. Presented results have been measured in two different OPLN in the city of Málaga: Pablo Ruíz Picasso avenue and SOHO district ${ }^{4}$. The latter is close to the city center, with straight streets and large buildings mainly occupied by offices and homes. The former location is in a residential district located by the seafront. Obtained results corroborated that, to a large extent, the conventional noise terms reported in broadband indoor PLC networks (periodic and sporadic impulsive noise, narrowband interference and background noise) are also present in OPLN [13]. 1) Description of the measurement set-up Two different set-ups have been employed. The first one is based on a computer equipped with a 12bit data-acquisition card with a sampling rate of 50 MSamples/s. This allows recording very long time domain registers for deeper analysis but its bandwidth is limited to $25 \mathrm{MHz}$. The input impedance can be set to $50 \Omega$ or $1 \mathrm{M} \Omega$. The second set-up is based on a

\footnotetext{
${ }^{4}$ See corresponding maps at https://goo.gl/8XzmZN and https://goo.gl/DeRqNA, respectively.
} 
spectrum analyzer with $3 \mathrm{GHz}$ bandwidth and $50 \Omega$ of input impedance. In both cases, adequate coupling circuits were used.

\section{2) Noise measurement results}

Fig. 8 shows the noise power spectral density (PSD) registered at four different points in the SOHO location: (1) corresponds to the control panel; (2) to the base of a lamp post with two lamps (of $250 \mathrm{~W}$ and $150 \mathrm{~W})$; while (3) and (4) have been taken at the base of two lamp posts with a lamp of $150 \mathrm{~W}$. As seen, noise level at the control panel is significantly higher than in the remaining points. Noise level at (2), where two lamps are connected, is also higher than in (3) and (4). Remarkable is the observed level of disturbance at the upper band, which corresponds to the ingress of interference from commercial FM radio stations (from 87.5 to $108 \mathrm{MHz}$ in Europe). Noise level at the control panel seems to be independent of the number of connected lamps, as well. This can be seen in Fig. 9, where the noise PSD measured at the control panels is depicted for two conditions: with just one phase and circuit switched on, and with all circuits and phases switched on. Finally, the existence of impulsive noise components that exhibit a cyclostationary behavior synchronous with the mains period (20 ms in Spain) is illustrated in Fig. 10.

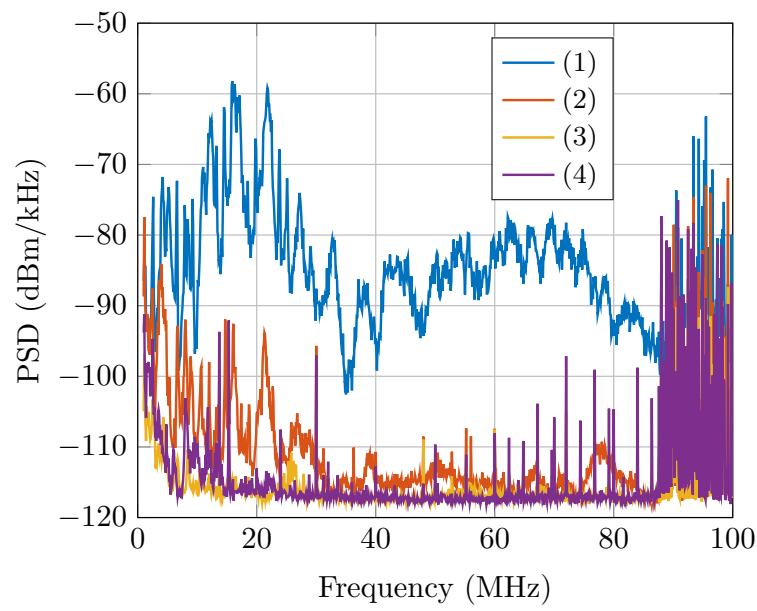

Fig. 8. Noise PSD estimated from measurements at the SOHO.

\section{SYSTEM PERFORMANCE ESTIMATIONS}

In this section, a statistical analysis of transmission system performance achieved over the set of modeled channel responses and the measurements of noise PSD is presented. A system simulator using orthogonal frequency division multiplexing (OFDM) has been implemented according to the parameters of the ITUT Rec. G.9960 standard [10], since it represents the state-of-the-art technology in PLC. Table I summarizes the main parameters. A PSD mask with a limit of $-20 \mathrm{dBm} / \mathrm{kHz}$ and $-50 \mathrm{dBm} / \mathrm{kHz}$ is applied to the transmitted signal in the band $2-30 \mathrm{Mz}$ and $30-80$ $\mathrm{MHz}$, respectively. It includes permanent frequency notches as well, which reduce the number of useful

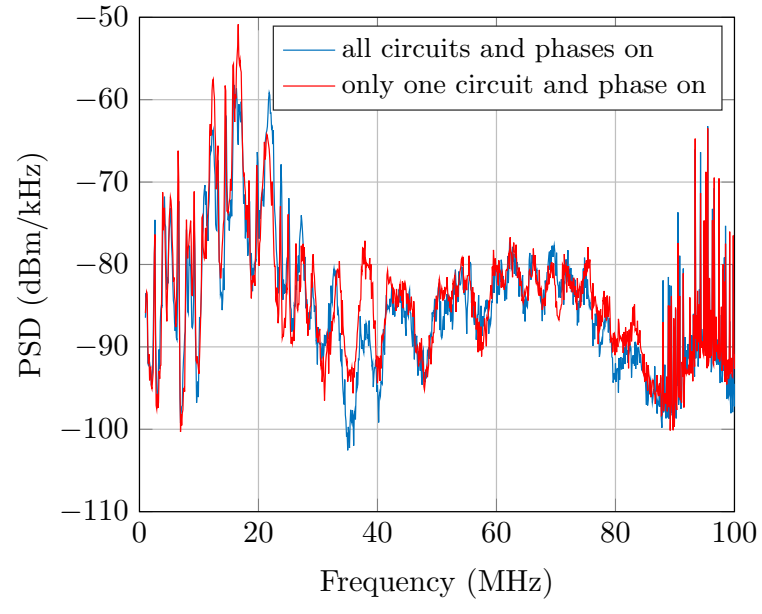

Fig. 9. Noise PSD at SOHO control panel for two conditions.

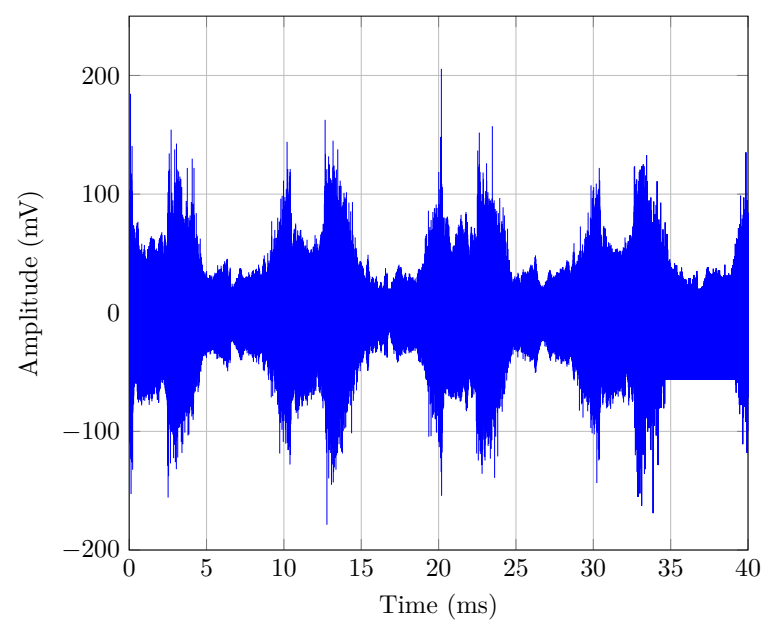

Fig. 10. Noise waveform exhibiting a cyclostationary behavior.

carriers to 3317 and yield a maximum physical layer bit-rate of $740.4 \mathrm{Mbit} / \mathrm{s}$.

TABLE I

SUMMARY OF THE EMPLOYED OFDM SYSTEM PARAMETERS.

\begin{tabular}{c|c} 
Parameter & Value \\
\hline frequency band & $2-80 \mathrm{MHz}$ \\
number of carriers & 4096 \\
carrier separation & $24.41 \mathrm{kHz}$ \\
cyclic prefix duration & $5.12 \mu \mathrm{s}$ \\
pulse-shaping extension & $5.12 \mu \mathrm{s}$ \\
constellation sizes & $1-12 \mathrm{bit} / \mathrm{symbol}$ \\
target bit error rate & $10^{-6}$ \\
code rate & $20 / 21$ \\
coding gain & $3 \mathrm{~dB}$ \\
system margin & $3 \mathrm{~dB}$ \\
\hline
\end{tabular}

Fig. 11 depicts a scatter plot of the estimated bit-rate vs link length for two noise conditions: a high noise scenario, obtained using the highest measured PSD, and a low noise one, computed with the lowest registered PSD. The bit-rate is always above $130 \mathrm{Mbit} / \mathrm{s}$ in links shorter than $50 \mathrm{~m}$. Additional details can be extracted from Fig. 12, where the complementary CDF 
(CCDF) of the bit-rate for different link distances is plotted. Recalling that bit-rates around $50 \mathrm{Mbit} / \mathrm{s}$ are currently required for small cells backhauling, it can be observed that this value is achieved for links shorter than $100 \mathrm{~m}$ in almost all cases. For link lengths of up to $150 \mathrm{~m}$, the referred bit-rate is achieved in $97 \%$ of the cases in the low noise scenario and in $85 \%$ of the cases in the high noise scenario. For link lengths up to $200 \mathrm{~m}$, the corresponding percentages are $88 \%$ and $64 \%$, respectively.

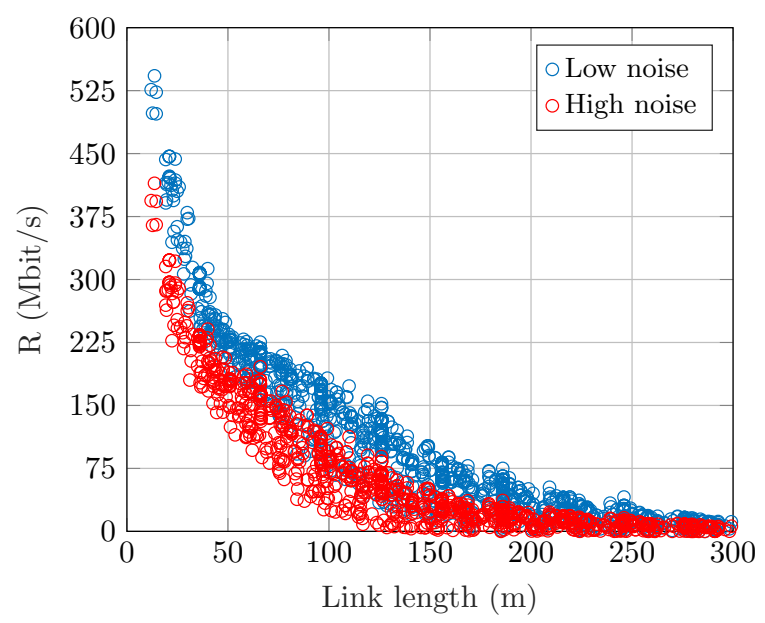

Fig. 11. Scatter plot of the bit-rate vs distance for two noise levels.

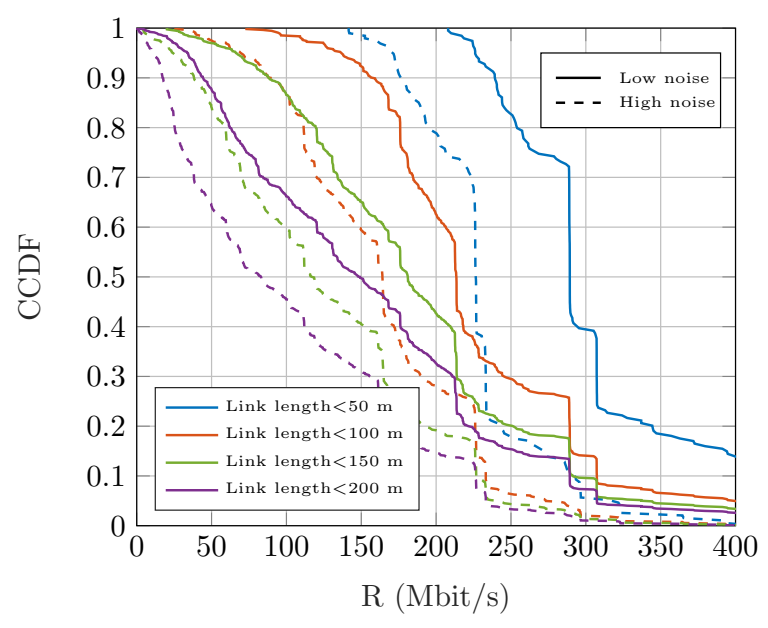

Fig. 12. CCDF of the bit-rate for different link lengths.

\section{Conclusion}

In this work, the use PLC over OPLN for small cells backhauling has been studied. The channel response of these kind of links has been modeled by means of a bottom-up simulator, from which hundreds of responses have been generated to perform a statistical analysis of their behavior. The simulation tool comprises two parts: one to model different network topologies based on their physical and electrical characteristics and another to calculate the channel frequency response of the links established in such networks. A measurement campaign in actual OPLN has been carried out to register realistic noise signals. The synthesized channel responses have been used together with the measured noise to estimate the performance of transmission systems according to the ITU-T Rec. G.9960 standard. Results indicate that the assessed PLC technology provides the required bit-rate for link lengths of up to $100 \mathrm{~m}$ even in worst-case noise conditions. For link lengths of up to $150 \mathrm{~m}$ the required bit-rate is attained in $85 \%$ of the cases. These figures can be considerably improved by incorporating the multiple-input multiple-output (MIMO) techniques defined in the ITU-T Rec. G.9963 standard, since four conductors are available at the base of the lamp post.

\section{ACKNOWLEDGMENT}

The authors thank Vodafone Group Services Limited, the Head of the Servicios Operativos of the City Council of Málaga, Manuel Salazar, and Monelec S.L. for the support provided to accomplish this work.

\section{REFERENCES}

[1] B. Rogers and G. Yeap, "The Emergence of LTE Small Cells and 5G," Cartesian: Specialist Consulting and Management Services, 2015. [On-line 30-11-2018]: https://goo.gl/KNXKEm

[2] A. Anpalagan, M. Bennis, R. Vannithamby (Eds.), "Design and Deployment of Small Cell Networks," Cambridge University Press, 2016.

[3] A. H. Jafari, D. López-Pérez, H. Song, H. Claussen, L. Ho, J. Zhang, "Small cell backhaul: challenges and prospective solutions", EURASIP Journal on Wireless Communications and Networking (2015:206), pp. 1-18, 2015.

[4] J. Edstam, "Microwave backhaul gets a boost with multiband," Ericcson Technology Review. pp. 1-11, January 2016.

[5] F. Marcuzzi, Andrea M. Tonello, "Stochastic Geometry for the Analysis of Small Radio Cells and PLC Back-Hauling," In Proceedings of the International ITG Conference on Systems, Communications and Coding, 2017.

[6] D. G. Kendall, "Branching Processes Since 1873," Journal of the London Mathematical Society, vol. 21-41, issue 1, pp. 385406, 1966.

[7] C. R. Paul, Analysis of Multiconductor Transmission Lines, Wiley, 1994.

[8] J. A. Corchado, J. A and Cortés, F. J. Cañete, L. Díez, "An MTL-Based Channel Model for Indoor Broadband MIMO Power Line Communications," IEEE Journal on Selected Areas in Communications, vol. 34, no. 7, pp. 2045-2055, July, 2016.

[9] J. A. Cortés, L. Díez, F. J. Cañete, J. L. González Moreno, "On the statistical properties of indoor power line channels: Measurements and models," in IEEE Int. Symp. on Power Line Communications and its Applications (ISPLC), March 2011.

[10] Unified high-speed wireline-based home networking transceivers - System architecture and physical layer specification, ITU-T Recommendation G.9960, July 2015.

[11] HomePlug AV White Paper, Homeplug, 2005.

[12] M. Zimmermann and K. Dostert, "Analysis and Modeling of Impulsive Noise in Broad-Band Powerline Communications," IEEE Transactions on Electromagnetic Compatibility, vol. 44, no. 1, pp. 249-258, Feb. 2002.

[13] J. A. Cortés, L. Díez, F. J. Cañete, J. J. Sánchez-Martínez, "Analysis of the Indoor Broadband Power-Line Noise Scenario," IEEE Transactions on Electromagnetic Compatibility, vol. 52, no. 4, pp. 849-858, Nov. 2010.

[14] M. Katayama, T. Yamazato, H. Okada, "A Mathematical Model of Noise in Narrowband Power Line Communication Systems," IEEE Journal on Selected Areas in Communications, vol. 24, no. 7, pp. 1267-1276, July 2006.

[15] J. A. Cortés, A. Sanz, P. Estopiñán, J. I. García, "Analysis of narrowband power line communication channels for advanced metering infrastructure," EURASIP Journal on Advances in Signal Processing, (2015:27), March 2015. 